\title{
Continuous Finite-Time Terminal Sliding Mode IDA-PBC Design for PMSM with the Port-Controlled Hamiltonian Model
}

\author{
Shuanghe Yu, Lina Jin, Kai Zheng, and Jialu Du \\ School of Information Science and Technology, Dalian Maritime University, Dalian 116026, China \\ Correspondence should be addressed to Shuanghe Yu; shuangyu@dlmu.edu.cn
}

Received 1 May 2013; Accepted 8 July 2013

Academic Editor: Yu Kang

Copyright ( 2013 Shuanghe Yu et al. This is an open access article distributed under the Creative Commons Attribution License, which permits unrestricted use, distribution, and reproduction in any medium, provided the original work is properly cited.

\begin{abstract}
Finite-time control scheme for speed regulation of permanent magnet synchronous motor (PMSM) is investigated under the portcontrolled Hamiltonian (PCH), terminal sliding mode (TSM), and fast TSM stabilization theories. The desired equilibrium is assigned to the PCH structure model of PMSM by maximum torque per ampere (MTPA) principle, and the desired Hamiltonian function of state error is constructed in the form of fractional power structure as TSM and fast TSM, respectively. Finite-time TSM and fast TSM controllers are designed via interconnection and damping assignment passivity-based control (IDA-PBC) methodology, respectively, and the finite-time stability of the desired equilibrium point is also achieved under the PCH framework. Simulation results validate the improved performance of the presented scheme.
\end{abstract}

\section{Introduction}

Recently, permanent magnet synchronous motors (PMSM) have become increasingly popular in high-performance AC drive applications because of their advantages over many other kinds of motors, such as induction motors and DC motors [1]. These high dynamical performances include high power density, torque-to-inertia ratio, and efficiency. However, the speed regulation of PMSM is a nonlinear control problem because of the strong coupling between the motor speed and the electrical quantities. Therefore, it is a challenging task to design a controller with highperformance of speed regulation.

Various nonlinear control theories have been investigated for the speed control of PMSM, such as sliding mode control [2], backstepping control [3], and predictive control [4]. Compared to these nonlinear controls, the energyshaping control can be implemented with a clear physical interpretation when a dynamical system is viewed as an energy transformation mechanism [5-8], for example, the port-controlled Hamiltonian (PCH) system [9]. A significant case of $\mathrm{PCH}$ is the port-controlled Hamiltonian system with dissipation (PCHD) expressed by its interconnection and damping structure with Hamiltonian energy function [10]. These explicit structural pieces of information naturally lead to the interconnection and damping assignment passivitybased control (IDA-PBC) framework [11-13]. The essence of IDA-PBC approach is to find a controller and an interconnection pattern such that the closed-loop system is preserved in a desired PCHD structure. The procedure sets up an easy way of stability analysis with the desired Hamiltonian energy function as the Lyapunov function. The speed regulation of PMSM has been investigated with IDA-PBC [14-16].

All these approaches share the common property of asymptotic stability with infinite convergent time. In contrast, the finite-time controller possesses not only fast convergence to the equilibrium states in finite-time but also stronger robustness and disturbance attenuation properties [17]. However, the finite-time control design is a challenging problem because such a controller leads to a non-Lipschitzian dynamics [18]. Finite-time control can be obtained in either a discontinuous or continuous manner. In practical implementations, the infinite fast switching of discontinuous control can lead to chattering behavior which may damage the actuators and excite unmodeled high-frequency dynamics. Continuous finite-time control can be obtained by fractional 
power feedback as the formalism of terminal sliding mode (TSM), resulting in a finite-time convergent closed-loop differential equation with fractional power. Higher order sliding mode control is also a kind of continuous finitetime control through using the discontinuous control in the higher order derivative of the control instead of the actual control. Furthermore, the speed regulation of PMSM has been investigated with the continuous finite-time controls $[19,20]$.

Based on a finite-time stability criterion and the energyshaping plus damping injection technique, the finite-time stabilization problem is investigated for the $\mathrm{PCH}$ systems $[21,22]$. According to the PCH structure model of PMSM, a kind of finite-time TSM feedback realization is proposed to achieve the rapid speed regulation of PMSM in this paper. The desired equilibrium is assigned by maximum torque per ampere (MTPA) principle, and the desired Hamiltonian function of state error is shaped in the form of fractional power structure. Finite-time TSM control is designed via IDA-PBC methodology.

The remaining part of the paper is organized as follows. Some background on the PCHD formalism and finite-time TSM stabilization principle is firstly introduced in Section 2. The problem is formulated in Section 3. These techniques are then applied to the speed regulation problem of PMSM in the form of PCH model, and the finite-time stability of equilibrium point is also given in Section 4. The closed-loop performance is evaluated via simulations in Section 5. Finally, some conclusions are presented in Section 6.

\section{Preliminaries}

2.1. Port-Controlled Hamiltonian with Dissipation (PCHD) Systems. PCHD system is a geometrically defined class of systems with an internal interconnection structure, a Hamiltonian function defined as the total stored energy, and a resistive structure. Energy dissipation is included in the framework of $\mathrm{PCH}$ systems by terminating some of the ports by resistive elements. PCHD systems are a class of passive systems, which have attracted the attention of many researchers lately, in particular for stabilization objectives. A PCHD system is defined as

$$
\begin{gathered}
\dot{x}=[J(x)-R(x)] \frac{\partial H}{\partial x}(x)+g(x) u, \\
y=g^{T}(x) \frac{\partial H}{\partial x}(x),
\end{gathered}
$$

where $x \in R^{n}$ is the state vector, $u, y \in R^{m}$ representing the input and output vector, respectively, are conjugated variables whose product represents the system environment power exchange, $H(x): R^{n} \rightarrow R$ is the stored energy function, $J(x)=-J^{T}(x): R^{n} \rightarrow R^{n \times n}$ captures the power-conserving interconnection structure, $R(x)=R^{T}(x) \geq 0: R^{n} \rightarrow R^{n \times n}$ is the dissipation matrix, and $g(x): R^{n} \rightarrow R^{n \times m}$ is the port matrix of the input $u$ on the system and also the output from the system via its transpose. In this case, the energy-balancing property takes the following form:

$$
\frac{d H}{d t}(x)=u^{T}(t) y(t)-\frac{\partial^{T} H}{\partial x}(x) R(x) \frac{\partial H}{\partial x}(x) \leq u^{T}(t) y(t),
$$

showing passivity if the Hamiltonian $H$ is bounded from below.

2.2. IDA-PBC Principle of PCHD Systems. The interconnection structural properties of PCHD systems can be exploited for the control design. While preserving the PCHD form, IDA-PBC technique can assign the desired interconnection matrix $J_{d}$ and dissipative matrix $R_{d}$ of the closed-loop and shape a new closed-loop Hamiltonian energy function $H_{d}$ with a stable desired equilibrium point. Consider the following:

$$
\dot{x}=\left[J_{d}(x)-R_{d}(x)\right] \frac{\partial H_{d}}{\partial x}(x) .
$$

Proposition 1. For system (1), given a desired equilibrium $x^{*}$, define the stabilization error as $\widetilde{x}=x-x^{*}$ and assign a closedloop energy function $H_{d}>0$ and $H_{d}=0$; the purpose of IDA$P B C$ is to find the new structure matrices $J_{a}$, damping matrix $R_{a}$, a vector function $K(x)$, and a feedback control $u=\beta(x)$ such that

$$
\begin{aligned}
{[J(x)} & \left.+J_{a}(x)-\left(R(x)-R_{a}(x)\right)\right] K(x) \\
& =-\left[J_{a}(x)-R_{a}(x)\right] \frac{\partial H}{\partial x}(x)+g(x) \beta(x),
\end{aligned}
$$

with the following properties.

(i) Structure preservation:

$$
\begin{gathered}
J_{d}(x)=J(x)+J_{a}(x)=-J_{d}^{T}(x), \\
R_{d}(x)=R(x)+R_{a}(x)=R_{d}^{T}(x)>0 .
\end{gathered}
$$

(ii) Integrability: $K(x)$ is the gradient of a scalar function. That is,

$$
\frac{\partial K}{\partial x}(x)=\frac{\partial^{T} K}{\partial x}(x)
$$

(iii) Equilibrium assignment: at the equilibrium $x^{*}, K(x)$ satisfies

$$
K\left(x^{*}\right)=-\frac{\partial H}{\partial x}\left(x^{*}\right) .
$$

(iv) Lyapunov stability: at the equilibrium $x^{*}$, the Jacobian of $K(x)$ satisfies the following bound:

$$
\frac{\partial K}{\partial x}\left(x^{*}\right)>-\frac{\partial^{2} H}{\partial x^{2}}\left(x^{*}\right) .
$$


Under these conditions, the closed-loop system will be a PCHD system with dissipation of the form (3) where

$$
\begin{gathered}
H_{d}(x)=H(x)+H_{a}(x), \\
\frac{\partial H_{a}}{\partial x}(x)=K(x) ;
\end{gathered}
$$

the desired equilibrium $x^{*}$ is asymptotically stable.

2.3. Finite-Time Stability of TSM. The TSM and fast TSM concepts are based on a class of nonlinear differential equations described by the following first order dynamics, respectively:

$$
\begin{gathered}
s=\dot{x}+\beta x^{\gamma}=0, \quad \beta>0,0<\gamma<1, \\
s=\dot{x}+\alpha x+\beta x^{\gamma}=0, \quad \alpha, \beta>0,0<\gamma<1 .
\end{gathered}
$$

Lemma 2. The equilibrium point $x=0$ of the continuous non-Lipschitz differential equations (10) is globally finite-time stable; that is, for any initial condition $x(0)=x_{0}$, the system state converges to $x=0$ in finite-time:

$$
\begin{gathered}
T\left(x_{0}\right)=\frac{1}{\beta(1-\gamma)}\left|x_{0}\right|^{1-\gamma}, \\
T\left(x_{0}\right)=\frac{1}{\alpha(1-\gamma)} \ln \frac{\alpha x_{0}^{1-\gamma}+\beta}{\beta},
\end{gathered}
$$

respectively, and stay there forever, that is, $x=0$ for $t>T\left(x_{0}\right)$.

A Lyapunov-type theorem has been developed for finitetime stability $[17,18]$.

Lemma 3. Consider the nonlinear system described in (1); suppose that there is a $C^{1}$ function $V(x)$ defined in a neighborhood $D \subset R^{n}$ of the origin, such that $V(x)>0$ on $D$ and

$$
\begin{gathered}
\dot{V}(x)+\beta V^{\gamma}(x) \leq 0, \\
\dot{V}(x)+\alpha V(x)+\beta V^{\gamma}(x) \leq 0
\end{gathered}
$$

along the trajectory on $D$. Then, the origin of the system is finitetime stable. Moreover, the settling time, depending on the initial state $x(0)=x_{0}$, is given by

$$
\begin{gathered}
T\left(x_{0}\right) \leq \frac{1}{\beta(1-\gamma)} V^{1-\gamma}\left(x_{0}\right), \\
T\left(x_{0}\right) \leq \frac{1}{\alpha(1-\gamma)} \ln \frac{\alpha x_{0}^{1-\gamma}+\beta}{\beta},
\end{gathered}
$$

for $x_{0}$ in some open neighborhood of the origin, respectively. If $D=R^{n}$ and $V(x)$ is also radially unbounded, the origin is globally finite-time stable.

2.4. Finite-Time Stability of PCHD System. Based on the finite-time convergence principle of TSM and FTSM, the corresponding finite-time stability of PCHD system can be summarized as follows $[21,22]$.
Lemma 4. Consider the PCHD system (3) with $J_{d}(x)=$ $-J_{d}^{T}(x)$ and $R_{d}(x)=R_{d}^{T}(x)>0$,

$$
\begin{gathered}
H_{d}(x)=\sum_{i=1}^{n} \beta_{i} x_{i}^{\gamma_{i}}, \\
H_{d}(x)=\frac{1}{2} \sum_{i=1}^{n} \alpha_{i} x_{i}^{2}+\sum_{i=1}^{n} \beta_{i} x_{i}^{\gamma_{i}},
\end{gathered}
$$

where $\alpha_{i}, \beta_{i}>0$ and $1<\gamma_{i}<2$; then, the equilibrium $x=0$ is globally finite-time stable.

\section{Problem Formulation}

The model of the PMSM can be described in a synchronously rotating $d-q$ reference frame as

$$
\begin{gathered}
L_{d} \frac{d i_{d}}{d t}=u_{d}-R_{s} i_{d}+n_{p} L_{q} i_{q} \omega, \\
L_{q} \frac{d i_{q}}{d t}=u_{q}-R_{s} i_{q}-n_{p} L_{d} i_{d} \omega-n_{p} \phi \omega, \\
J \frac{d \omega}{d t}=\tau-\tau_{L}=n_{p} \phi i_{q}-\tau_{L},
\end{gathered}
$$

where $D=\operatorname{diag}\left\{L_{d} L_{q} J\right\}, L_{d}=L_{q}, n_{p}$ is the number of pole pairs, $\omega$ is the mechanical angular speed of the rotor, $J$ is the moment of inertia, $L_{d}$ and $L_{q}$ are $d$-axis and $q$-axis stator inductances, respectively, $R$ is the stator resistance per phase, $\tau$ and $\tau_{L}$ are the electromagnetic and load torque, respectively, and $\phi$ is the rotor flux linking the stator.

The PMSM model (16) can be written as a PCHD system as follows:

$$
\begin{gathered}
\dot{x}=[J(x)-R(x)] \frac{\partial H}{\partial x}(x)+g(x) u, \\
y=g^{T}(x) \frac{\partial H}{\partial x}(x),
\end{gathered}
$$

where the state, input, and output are defined as follows, respectively:

$$
\begin{gathered}
x=\left[\begin{array}{l}
x_{1} \\
x_{2} \\
x_{3}
\end{array}\right]=\left[\begin{array}{ccc}
L_{d} & 0 & 0 \\
0 & L_{q} & 0 \\
0 & 0 & J
\end{array}\right]\left[\begin{array}{l}
i_{d} \\
i_{q} \\
\omega
\end{array}\right]=D\left[\begin{array}{l}
i_{d} \\
i_{q} \\
\omega
\end{array}\right] \\
u=\left[\begin{array}{lll}
u_{d} & u_{q}-\tau_{L}
\end{array}\right]^{T}, \quad y=\left[\begin{array}{lll}
i_{d} & i_{q} & \omega
\end{array}\right]^{T},
\end{gathered}
$$

and the interconnection, dissipative, weight, and Hamiltonian matrix are defined as follows, respectively:

$$
\begin{gathered}
J(x)=\left[\begin{array}{ccc}
0 & 0 & n_{p} x_{2} \\
0 & 0 & -n_{p}\left(x_{1}+\phi\right) \\
-n_{p} x_{2} & n_{p}\left(x_{1}+\phi\right) & 0
\end{array}\right], \\
R=\left[\begin{array}{ccc}
R_{s} & 0 & 0 \\
0 & R_{s} & 0 \\
0 & 0 & 0
\end{array}\right], \quad g=\left[\begin{array}{lll}
1 & 0 & 0 \\
0 & 1 & 0 \\
0 & 0 & 1
\end{array}\right], \\
H(x)=\frac{1}{2}\left[\frac{1}{L_{d}} x_{1}^{2}+\frac{1}{L_{q}} x_{2}^{2}+\frac{1}{J} x_{3}^{2}\right] .
\end{gathered}
$$


According to the principle of maximum torque per ampere, the desired equilibrium can be acquired as

$$
x^{*}=\left[\begin{array}{lll}
x_{1}^{*} & x_{2}^{*} & x_{3}^{*}
\end{array}\right]=\left[\begin{array}{lll}
0 & \frac{L_{q} \tau_{L}}{n_{p} \phi} & J \omega^{*}
\end{array}\right],
$$

where $\tau_{L}$ and $\omega^{*}$ are the known load torque and references speed, respectively.

The control objective is to design a state-feedback control $u=u(x)$ such that the closed-loop dynamics is a PCHD form (3) with the strict local minimum at the desired equilibrium $x^{*}$ as (20), and the equilibrium is finite-time stable.

\section{Finite-Time TSM Controller Design via IDA-PBC}

According to the desired equilibrium (20), the desired Hamiltonian function is chosen as

$$
\begin{array}{r}
H_{d}(x)=\frac{1}{\gamma+1}\left(\frac{1}{L_{d}}\left(x_{1}-x_{1}^{*}\right)^{\gamma+1}+\frac{1}{L_{q}}\left(x_{2}-x_{2}^{*}\right)^{\gamma+1}\right. \\
\left.+\frac{1}{J}\left(x_{3}-x_{3}^{*}\right)^{\gamma+1}\right), \quad 0<\gamma<1 ;
\end{array}
$$

IDA-PBC approach first designs the desired structure of interconnection and damping matrices named as IDA. Then, we derive a PDE parameterized by the chosen matrices whose solutions characterize all the energy functions that can be assigned. Finally, from this family of solutions, we choose one that satisfies the minimum requirement and computes the control. More precisely, the final objective of IDA-PBC is to find a static state-feedback control such that the closedloop dynamics is a desired PCH system with dissipation of the form (3).

We choose

$$
J_{a}(x)=\left[\begin{array}{ccc}
0 & -J_{12} & J_{13} \\
J_{12} & 0 & -J_{23} \\
-J_{13} & J_{23} & 0
\end{array}\right], \quad R_{a}=\left[\begin{array}{ccc}
r_{1} & 0 & 0 \\
0 & r_{2} & 0 \\
0 & 0 & 0
\end{array}\right],
$$

where $J_{12}, J_{13}, J_{23}, r_{1}, r_{2}$ are the interconnection and damping parameters to be designed and $r_{1}, r_{2}>0$. According to the standard procedure of IDA-PBC [12], we have

$$
\begin{aligned}
& J_{d}(x)=J(x)+J_{a}(x) \\
&=\left[\begin{array}{ccc}
0 & -J_{12} & J_{13}+n_{p} x_{2} \\
J_{12} & 0 & -J_{23}-n_{p}\left(x_{1}+\phi\right) \\
-J_{13}-n_{p} x_{2} & J_{23}+n_{p}\left(x_{1}+\phi\right) & 0
\end{array}\right], \\
& R_{d}(x)=R(x)+R_{a}(x)=\left[\begin{array}{ccc}
R_{s}+r_{1} & 0 & 0 \\
0 & R_{s}+r_{2} & 0 \\
0 & 0 & 0
\end{array}\right],
\end{aligned}
$$

$$
\begin{aligned}
& J_{d}(x)-R_{d}(x) \\
& \quad=\left[\begin{array}{ccc}
-\left(R_{s}+r_{1}\right) & -J_{12} & J_{13}+n_{p} x_{2} \\
J_{12} & -\left(R_{s}+r_{2}\right) & -J_{23}-n_{p}\left(x_{1}+\phi\right) \\
-J_{13}-n_{p} x_{2} & J_{23}+n_{p}\left(x_{1}+\phi\right) & 0
\end{array}\right],
\end{aligned}
$$

$$
\begin{aligned}
J_{a}(x)-R_{a}=\left[\begin{array}{ccc}
-r_{1} & -J_{12} & J_{13} \\
J_{12} & -r_{2} & -J_{23} \\
-J_{13} & J_{23} & 0
\end{array}\right], \\
K(x)=\frac{\partial H_{d}}{\partial x}-\frac{\partial H}{\partial x} \\
=\left[\frac{1}{L_{d}}\left(x_{1}-x_{1}^{*}\right)^{\gamma}, \frac{1}{L_{q}}\left(x_{2}-x_{2}^{*}\right)^{\gamma}, \frac{1}{J}\left(x_{3}-x_{3}^{*}\right)^{\gamma}\right]^{T} \\
= \\
=\left[\frac{1}{L_{d}} x_{1}, \frac{1}{L_{q}} x_{2}, \frac{1}{J} x_{3}\right]^{T} \\
{\left[\left(x_{1}-x_{1}^{*}\right)^{\gamma}-x_{1}\right], \frac{1}{L_{q}}\left[\left(x_{2}-x_{2}^{*}\right)^{\gamma}-x_{2}\right], } \\
\left.\frac{1}{J}\left[\left(x_{3}-x_{3}^{*}\right)^{\gamma}-x_{3}\right]\right]^{T} .
\end{aligned}
$$

Through solving the following matched equation,

$$
\begin{aligned}
{\left[J_{d}(x)\right.} & \left.-R_{d}(x)\right] K(x) \\
& =-\left[J_{a}(x)-R_{a}(x)\right] D^{-1} x+g(x) u(x) .
\end{aligned}
$$

The controller $u(x)=\left[\begin{array}{lll}u_{d} & u_{q} & -\tau_{L}\end{array}\right]^{T}$ can be obtained as

$$
\begin{aligned}
u_{d}= & -r_{1} \frac{x_{1}}{L_{d}}-\frac{J_{12} x_{2}}{L_{q}}+\frac{J_{13} x_{3}}{J}-\frac{R_{s}+r_{1}}{L_{d}} \\
& \times\left[\left(x_{1}-x_{1}^{*}\right)^{\gamma}-x_{1}\right]-\frac{J_{12}}{L_{q}}\left[\left(x_{2}-x_{2}^{*}\right)^{\gamma}-x_{2}\right] \\
& +\frac{J_{13}+n_{p} x_{2}}{J}\left[\left(x_{3}-x_{3}^{*}\right)^{\gamma}-x_{3}\right], \\
u_{q}= & \frac{J_{12} x_{1}}{L_{d}}-\frac{r_{2} x_{2}}{L_{q}}-\frac{J_{23} x_{3}}{J}+\frac{J_{12}}{L_{d}}\left[\left(x_{1}-x_{1}^{*}\right)^{\gamma}-x_{1}\right] \\
& -\frac{R_{s}+r_{2}}{L_{q}}\left[\left(x_{2}-x_{2}^{*}\right)^{\gamma}-x_{2}\right] \\
& -\frac{J_{23}+n_{p}\left(x_{1}+\phi\right)}{J}\left[\left(x_{3}-x_{3}^{*}\right)^{\gamma}-x_{3}\right] \\
= & \frac{J_{12} x_{1}}{L_{d}}-\frac{r_{2} x_{2}}{L_{q}}-\frac{J_{23} x_{3}}{J}+\frac{J_{12}}{L_{d}}\left[\left(x_{1}-x_{1}^{*}\right)^{\gamma}-x_{1}\right] \\
& -\frac{R_{s}+r_{2}}{L_{q}}\left[\left(x_{2}-x_{2}^{*}\right)^{\gamma}-x_{2}\right] \\
& -\frac{J_{23}+n_{p}\left(x_{1}+\phi\right)}{J}\left(x_{3}-x_{3}^{*}\right)^{\gamma} \\
& +\frac{J_{23} x_{3}}{J}+\frac{n_{p}\left(x_{1}+\phi\right) x_{3}}{J}
\end{aligned}
$$




$$
\begin{aligned}
= & \frac{J_{12} x_{1}}{L_{d}}-\frac{r_{2} x_{2}}{L_{q}}+\frac{J_{12}}{L_{d}}\left[\left(x_{1}-x_{1}^{*}\right)^{\gamma}-x_{1}\right] \\
& -\frac{R_{s}+r_{2}}{L_{q}}\left[\left(x_{2}-x_{2}^{*}\right)^{\gamma}-x_{2}\right] \\
& -\frac{J_{23}+n_{p}\left(x_{1}+\phi\right)}{J}\left(x_{3}-x_{3}^{*}\right)^{\gamma} \\
& +\frac{n_{p}\left(x_{1}+\phi\right) x_{3}}{J}, \\
-\tau_{L}= & -\frac{J_{13}}{L_{d}} x_{1}+\frac{J_{23}}{L_{q}} x_{2}-\frac{J_{13}+n_{p} x_{2}}{L_{d}} \\
& \times\left(\left(x_{1}-x_{1}^{*}\right)^{\gamma}-x_{1}\right)+\frac{J_{23}+n_{p}\left(x_{1}+\phi\right)}{L_{q}} \\
& \times\left(\left(x_{2}-x_{2}^{*}\right)^{\gamma}-x_{2}\right) \\
= & -\tau_{L}-\frac{J_{13}+n_{p} x_{2}}{L_{d}}\left(x_{1}-x_{1}^{*}\right)^{\gamma}+\frac{J_{23}}{L_{q}}\left(x_{2}-x_{2}^{*}\right)^{\gamma} \\
& +\left(\frac{J_{23}}{L_{q}}+\frac{n_{p}\left(x_{1}+\phi\right)}{L_{q}}-\frac{n_{p} \phi}{L_{q}}\left(x_{2}-x_{2}^{*}\right)^{1-\gamma}\right) \\
& +\frac{n_{p}\left(x_{1}+\phi\right)}{L_{q}}\left(x_{2}-x_{2}^{*}\right)^{\gamma}-\frac{n_{p} \phi}{L_{q}}\left(x_{2}-x_{2}^{*}\right) \\
& -\frac{J_{13}+n_{p} x_{2}}{L_{d}}\left(x_{1}-x_{1}^{*}\right)^{\gamma} \\
& \\
&
\end{aligned}
$$

In this paper, we choose the free parameters $J_{12}, J_{13}, J_{23}$ as

$$
\begin{gathered}
J_{12}=0, \quad J_{13}=-n_{p} x_{2}, \\
J_{23}=-n_{p}\left(x_{1}+\phi\right)+n_{p} \phi\left(x_{2}-x_{2}^{*}\right)^{1-\gamma} .
\end{gathered}
$$

Then, the final finite-time TSM controller $u(x)$ becomes

$$
\begin{gathered}
u_{d}=-r_{1} i_{d}-n_{p} L_{q} i_{q} \omega-\left(R_{s}+r_{1}\right)\left[L_{d}^{\gamma-1} i_{d}^{\gamma}-i_{d}\right], \\
u_{q}=-r_{2} i_{q}+n_{p} \omega\left(L_{d} i_{d}+\phi\right)-\frac{R_{s}+r_{2}}{L_{q}}
\end{gathered}
$$

$$
\begin{aligned}
& \times\left[\left(L_{q} i_{q}-\frac{L_{q} \tau_{L}}{n_{p} \phi}\right)^{\gamma}-L_{q} i_{q}\right] \\
& -\frac{n_{p} \phi\left(L_{q} i_{q}-\left(L_{q} \tau_{L} / n_{p} \phi\right)\right)^{1-\gamma}}{J}\left(J \omega-J \omega^{*}\right)^{\gamma}
\end{aligned}
$$$$
-\tau_{L}=-\tau_{L} .
$$

By applying this control to the PMSM system (16), the desired closed-loop PCHD system (3) can be reached with the desired hamiltonian function (21). With Lemma 4, the finite-time stability of the equilibrium (20) can be obtained.

Remark 5. Actually, the desired hamiltonian function (21) can be rewritten as

$$
\begin{aligned}
H_{d}(x)= & \frac{1}{\gamma+1} \\
& \times\left(\frac{1}{L_{d}}\left(\left(x_{1}-x_{1}^{*}\right)^{2}\right)^{(\gamma+1) / 2}+\frac{1}{L_{q}}\left(\left(x_{2}-x_{2}^{*}\right)^{2}\right)^{(\gamma+1) / 2}\right. \\
& \left.\quad+\frac{1}{J}\left(\left(x_{3}-x_{3}^{*}\right)^{2}\right)^{(\gamma+1) / 2}\right) .
\end{aligned}
$$

Because of $0<(\gamma+1) / 2<1$, according to Theorem 1 in [21], the closed-loop system will converge to the desired equilibrium (20) in finite-time.

Remark 6. In the controller (27), all the fractional powers of the state variables are positive; therefore, the singularity problem with conventional TSM controller is avoided.

Remark 7. When $\gamma=1$, the controller (27) is reduced to the conventional IDA-PBC design as in [14, 16]:

$$
\begin{gathered}
u_{d}=-r_{1} i_{d}-n_{p} L_{q} i_{q} \omega \\
u_{q}=-r_{2}\left(i_{q}-\frac{\tau^{*}}{n_{p} \phi}\right)+n_{p} \omega L_{d} i_{d}+\frac{R_{s}}{n_{p} \phi} \tau^{*}+n_{p} \phi \omega^{*} .
\end{gathered}
$$

Remark 8. If we choose the desired hamiltonian function as

$$
\begin{aligned}
& H_{d}(x)=\frac{1}{2}( \frac{1}{L_{d}}\left(x_{1}-x_{1}^{*}\right)^{2}+\frac{1}{L_{q}}\left(x_{2}-x_{2}^{*}\right)^{2} \\
&+\left.\frac{1}{J}\left(x_{3}-x_{3}^{*}\right)^{2}\right) \\
&+\frac{1}{\gamma+1}( \frac{1}{L_{d}}\left(x_{1}-x_{1}^{*}\right)^{\gamma+1}+\frac{1}{L_{q}}\left(x_{2}-x_{2}^{*}\right)^{\gamma+1} \\
&\left.+\frac{1}{J}\left(x_{3}-x_{3}^{*}\right)^{\gamma+1}\right),
\end{aligned}
$$




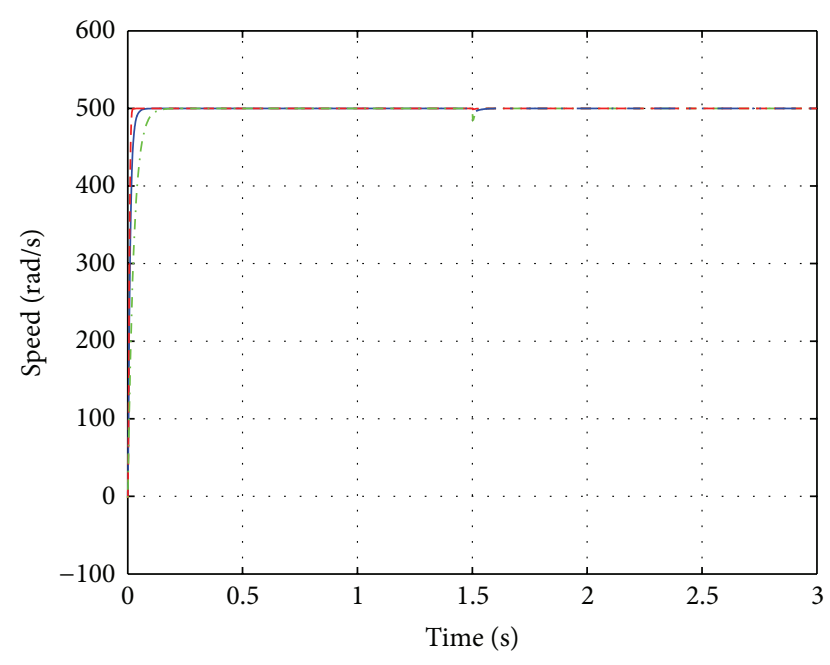

FIgURE 1: The speed response with different control strategies.

following the similar IDA-PBC procedure as before, we can design the fast TSM controller as

$$
\begin{gathered}
u_{d}=-r_{1} i_{d}-n_{p} L_{q} i_{q} \omega-\frac{R_{s}+r_{1}}{L_{d}}\left(L_{d} i_{d}\right)^{\gamma} \\
u_{q}=-\frac{r_{2} x_{2}}{L_{q}}-\frac{R_{s}+r_{2}}{L_{q}}\left[\left(x_{2}-x_{2}^{*}\right)^{\gamma}-x_{2}^{*}\right]+\frac{n_{p} x_{1} x_{3}}{J} \\
+\frac{n_{p} \phi x_{3}-n_{p} \phi\left(x_{2}-x_{2}^{*}\right)^{1-\gamma}\left(x_{3}-x_{3}^{*}\right)^{\gamma}+n_{p} \phi\left(x_{2}-x_{2}^{*}\right)^{1-\gamma} x_{3}^{*}}{J\left(1+\left(x_{2}-x_{2}^{*}\right)^{1-\gamma}\right)} .
\end{gathered}
$$

When $\gamma=1$, the previous controller is also reduced to the conventional IDA-PBC as

$$
\begin{gathered}
u_{d}=-\left(R_{s}+2 r_{1}\right) i_{d}-n_{p} L_{q} i_{q} \omega \\
u_{q}=-\left(R_{s}+2 r_{2}\right)\left(i_{q}-\frac{\tau^{*}}{n_{p} \phi}\right)+n_{p} \omega L_{d} i_{d} \\
+\frac{R_{s}}{n_{p} \phi} \tau^{*}+n_{p} \phi \omega^{*} .
\end{gathered}
$$

\section{Simulation Results}

In this section, the speed regulation performance of the PMSM based on the proposed controllers is investigated. The system parameters are $R_{s}=2.875 \Omega, \tau_{L}=1 \mathrm{~N} \cdot \mathrm{m}, J=$ $0.00085 \mathrm{~kg} \cdot \mathrm{m}^{2}, n_{p}=4, \phi=0.175 \mathrm{wb}$, and $L_{d}=L_{q}=$ $0.0085 \mathrm{H}$. The reference speed is $500 \mathrm{rad} / \mathrm{s}$. The initial load torque is $1 \mathrm{~N} \cdot \mathrm{m}$ and becomes $2 \mathrm{~N} \cdot \mathrm{m}$ at $t=1.5 \mathrm{~s}$.

The simulation results are shown in Figures 1, 2, 3, 4, 5, and 6. Figure 1 illustrates the speed response with different control strategies: fast TSM control (31) (red dash line), TSM control (27) (blue real line), and conventional IDA-PBC (29) (green dash dot line). The comparison results demonstrate that the proposed control strategies have superior performance on rapid response and disturbance rejection with

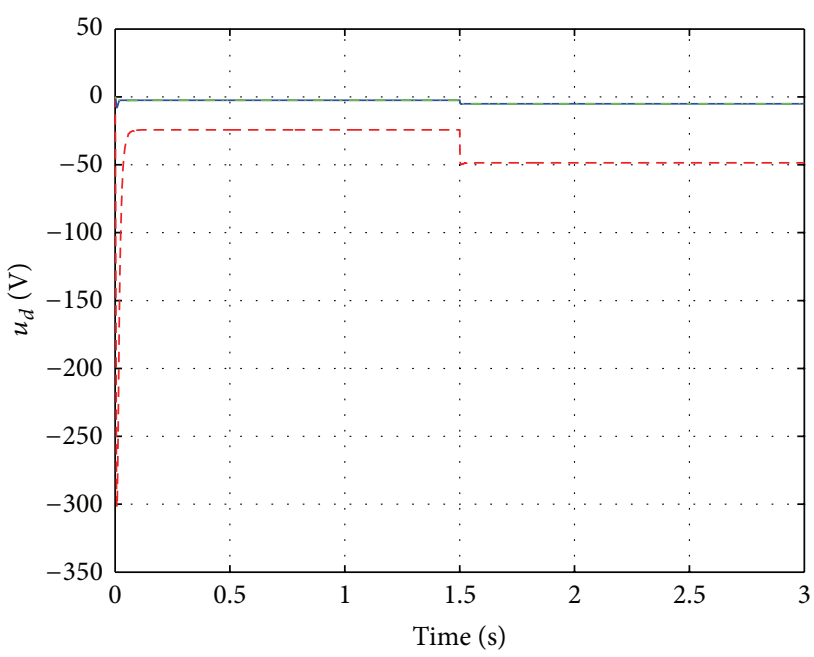

FIgURE 2: The $d$-axis control voltage.

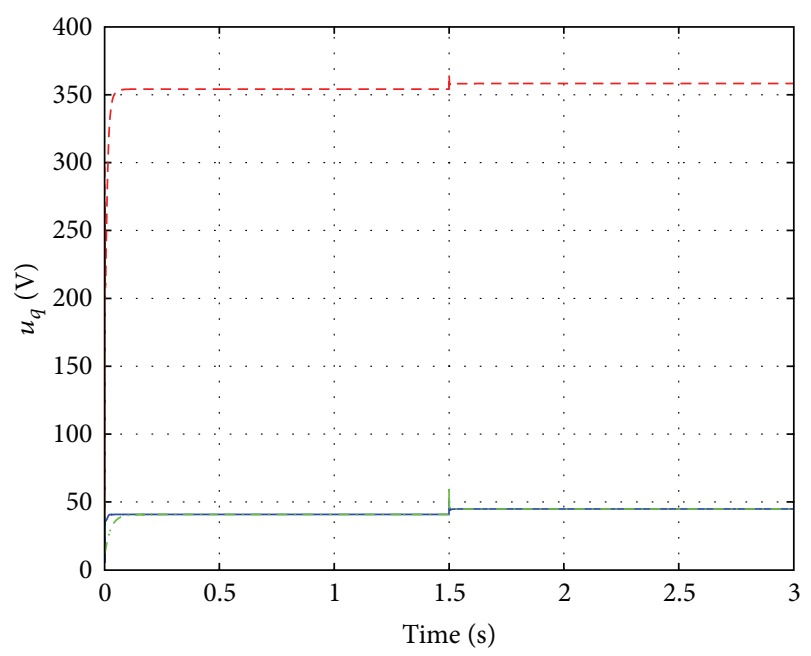

FIgURE 3: The $q$-axis control voltage.

respect to the conventional IDA-PBC. The speed regulation can reach the reference speed in finite-time $0.02 \mathrm{~s}$ and $0.12 \mathrm{~s}$ for fast TSM control and TSM control, respectively, and the conventional IDA-PBC only obtains the asymptotical convergence to the reference speed. When the load perturbance happens at $t=1.5 \mathrm{~s}$, the maximum speed variations are 499 , 494 , and $486 \mathrm{rad} / \mathrm{s}$ for the three controllers, respectively. The speed tracking performance is improved greatly as shown in Figure 1. This is because the control current, voltage, and torque response can rapidly change to deal with reference speed and load torque variation with the proposed control strategies as shown in Figures 2-6.

\section{Conclusions}

This paper studies the finite-time speed regulating problem of PMSM with TSM and PCHD theories. A fractional power form controller is designed with finite-time convergence to 


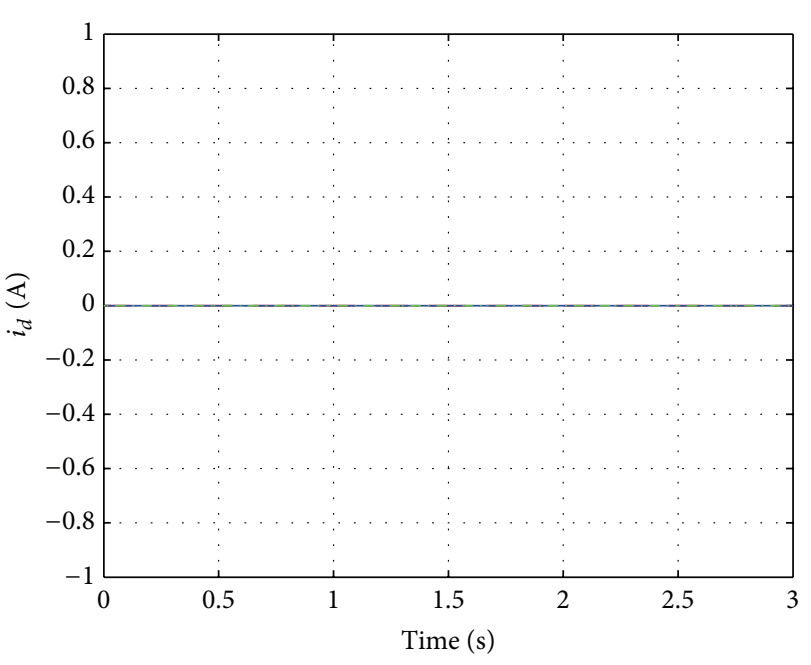

FIgURE 4: The $d$-axis control current.

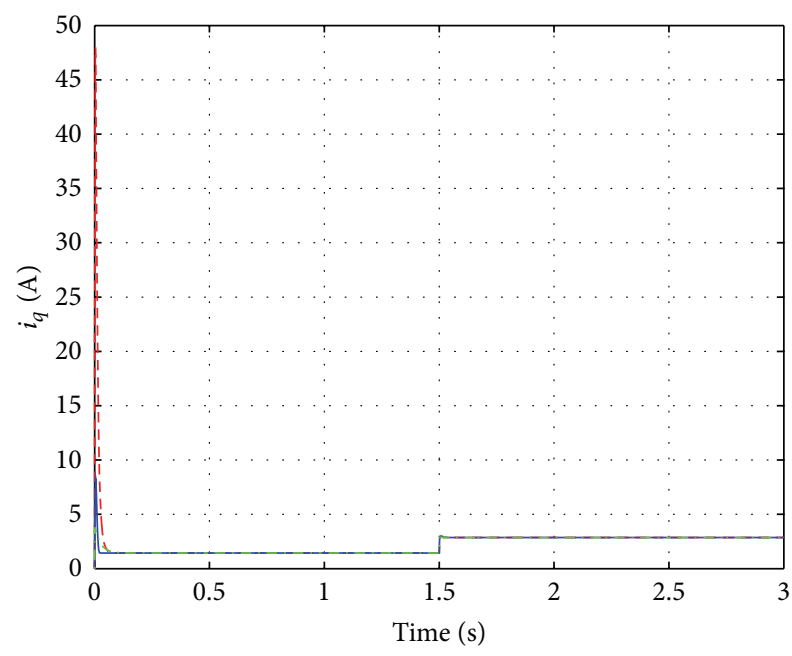

FIGURE 5: The $q$-axis control current.

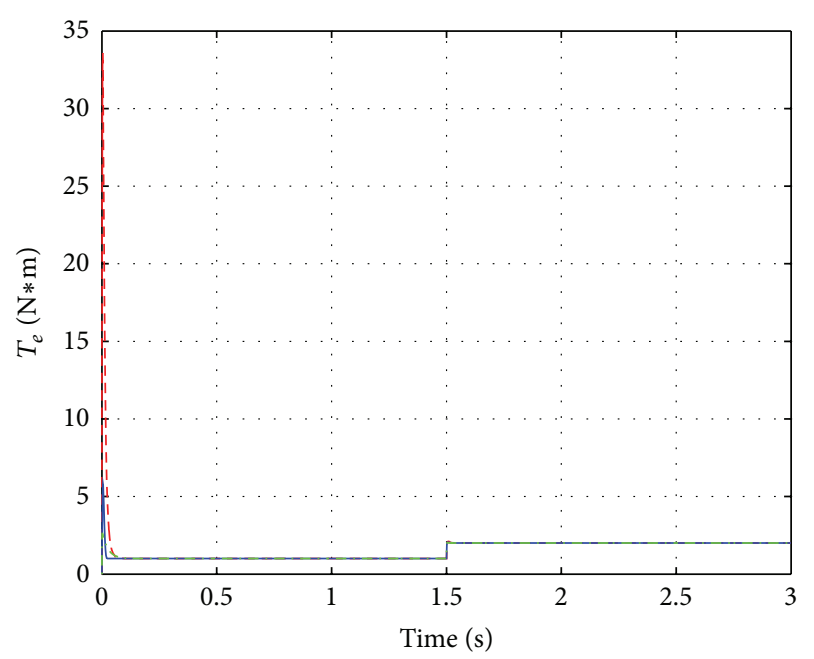

Figure 6: The control torque. the desired equilibrium. By comparing finite-time stable system with asymptotically stable system, the simulation results show that finite-time control method has faster convergence, better robustness, and antidisturbance, which improves the permanent magnet synchronous motor vector control system of performance. Further research will conduct the finite-time observer and finite-time disturbance observer and combine them with the current results.

\section{Acknowledgments}

This work is supported by the Doctoral Fund of Ministry of Education of China (no. 20102125120001), the Chinese National Science Foundation (nos. 60736022 and 51079013), and the Liaoning BaiQianWan Talents Program (2012921079).

\section{References}

[1] M. E. Haque, Permanent Magnet Synchronous Motor Drives: Analysis, Modeling and Control, VDM Verlag, 2009.

[2] S.-H. Chang, P.-Y. Chen, Y.-H. Ting, and S.-W. Hung, "Robust current control-based sliding mode control with simple uncertainties estimation in permanent magnet synchronous motor drive systems," IET Electric Power Applications, vol. 4, no. 6, pp. 441-450, 2010.

[3] J. Zhou and Y. Wang, "Adaptive backstepping speed controller design for a permanent magnet synchronous motor," IEE Proceeding Electric Power Applications, vol. 149, no. 2, pp. 165172, 2002.

[4] F. Morel, X. Lin-Shi, J.-M. Rtif, B. Allard, and C. Buttay, "A comparative study of predictive current control schemes for a permanent-magnet synchronous machine drive," IEEE Transactions on Industrial Electronics, vol. 56, no. 7, pp. 2715$2728,2009$.

[5] R. Ortega, A. J. van der Schaft, I. Mareels, and B. Maschke, "Putting energy back in control," IEEE Control Systems Magazine, vol. 21, no. 2, pp. 18-33, 2001.

[6] M. Galaz, R. Ortega, A. S. Bazanella, and A. M. Stankovic, "An energy-shaping approach to the design of excitation control of synchronous generators," Automatica, vol. 39, no. 1, pp. 111-119, 2003.

[7] D. E. Chang, "Some results on stabilizability of controlled lagrangian systems by energy shaping," in Proceedings of the IFAC World Congress, pp. 3161-3166, Seoul, Korea, 2008.

[8] W. M. Haddad, S. G. Nersesov, and V. Chellaboina, "Energybased control for hybrid port-controlled Hamiltonian systems," Automatica, vol. 39, no. 8, pp. 1425-1435, 2003.

[9] A. Venkatraman and A. J. van der Schaft, "Energy shaping of port-Hamiltonian systems by using alternate passive outputs," in Proceedings of the European Control Conference, pp. 21752180, Budapest, Hungary, 2009.

[10] B. Maschke, R. Ortega, and A. J. van der Schaft, "Energybased Lyapunov functions for forced Hamiltonian systems with dissipation," IEEE Transactions on Automatic Control, vol. 45, no. 8, pp. 1498-1502, 2000.

[11] R. Ortega, A. J. van der Schaft, B. M. Maschke, G. Escobar, and Interconnection, "and damping assignment passivity-based control: a survey," European Journal of Control, vol. 10, no. 5, pp. 432-450, 2004. 
[12] R. Ortega, A. van der Schaft, B. Maschke, and G. Escobar, "Interconnection and damping assignment passivity-based control of port-controlled Hamiltonian systems," Automatica, vol. 38, no. 4, pp. 585-596, 2002.

[13] S.-C. Lee and J. H. Park, "Performance improvement of PI controller with nonlinear error shaping function: IDA-PBC approach," Applied Mathematics and Computation, vol. 215, no. 10, pp. 3620-3630, 2010.

[14] V. Petrovic, R. Ortega, and A. tankovic, "Interconnection and damping assignment approach to control of permanent magnet synchrnous motor," IEEE Transactions on Control System Technology, vol. 9, no. 6, pp. 811-820, 2001.

[15] Y. Guo, Z. Xi, and D. Cheng, "Speed regulation of permanent magnet synchronous motor via feedback dissipative Hamiltonian realisation," IET Control Theory \& Applications, vol. 1, no. 1, pp. 281-290, 2007.

[16] H. Yu, Z. Zou, and S. Yu, "Speed regulation of PMSM based on port-controlled hamiltonian systems and PI control principle," in Proceedings of IEEE International Conference on Automation and Logistics, pp. 647-651, 2009.

[17] S. H. Yu, X. Yu, B. Shirinzadeh, and Z. Man, "Continuous finitetime control for robotic manipulators with terminal sliding mode," Automatica, vol. 41, no. 11, pp. 1957-1964, 2005.

[18] S. P. Bhat and D. S. Bernstein, "Finite-time stability of continuous autonomous systems," SIAM Journal on Control and Optimization, vol. 38, no. 3, pp. 751-766, 2000.

[19] S. H. Li, H. Liu, and S. Ding, "A speed control for a PMSM using finite-time feedback control and disturbance compensation," Transactions of the Institute of Measurement and Control, vol. 32, no. 2, pp. 170-187, 2010.

[20] S. Laghrouche, F. Plestan, and A. Glumineau, "A higher order sliding mode controller for a class of MIMO nonlinear systems: application to PM synchronous motor control," in Proceedings of American Control Conference, pp. 2592-2597, Boston, Mass, USA, 2004.

[21] Y. Z. Wang and G. Feng, "Finite-time stabilization of portcontrolled Hamiltonian systems with application to nonlinear affine systems," in Proceedings of American Control Conference, pp. 1202-1207, 2008.

[22] S. M. Ma and Y. Z. Wang, "Finite-time stability of a class of generalized Hamiltonian systems with application to control design of nonlinear affine systems," Journal of Shandong University, vol. 41, no. 2, pp. 119-125, 2011. 


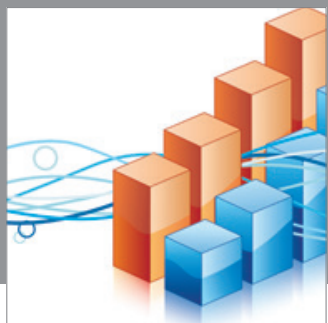

Advances in

Operations Research

mansans

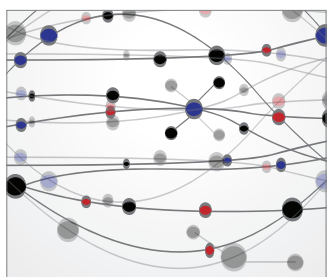

The Scientific World Journal
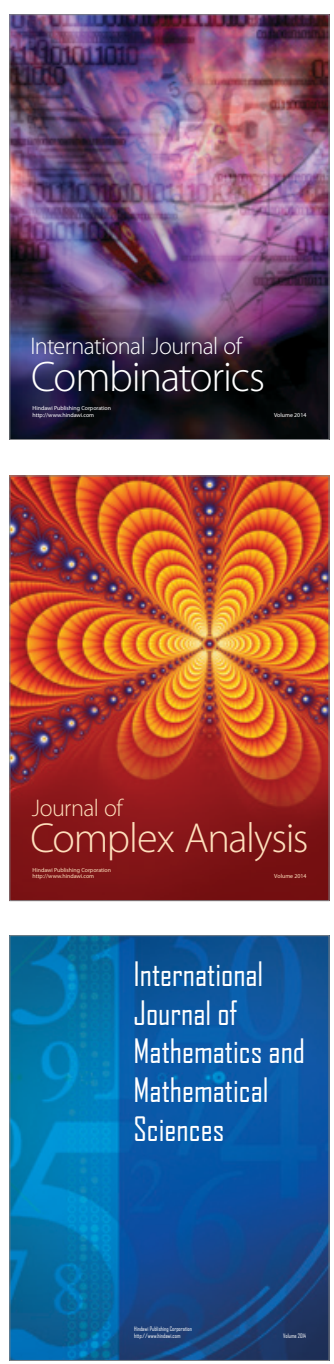
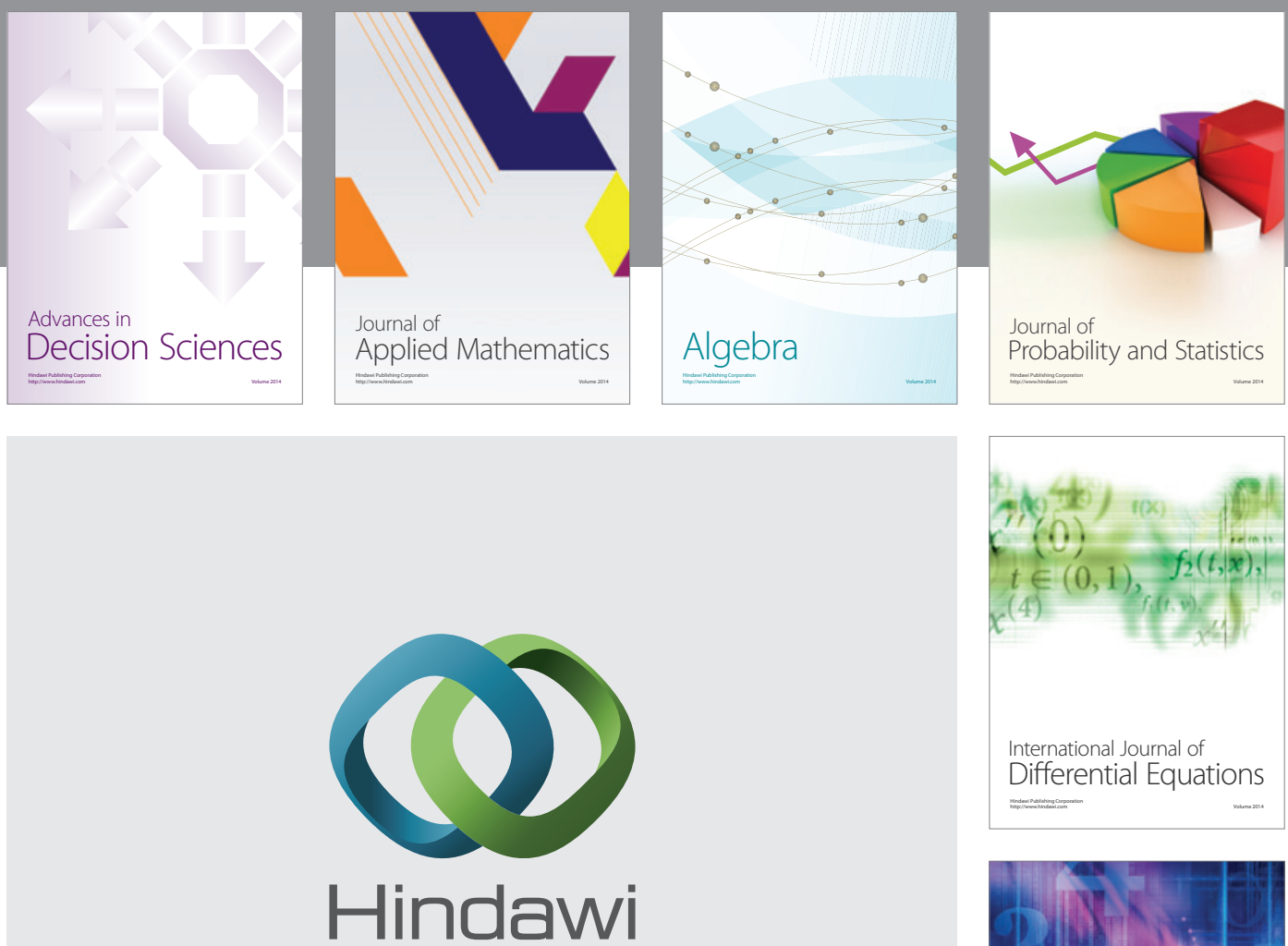

Submit your manuscripts at http://www.hindawi.com
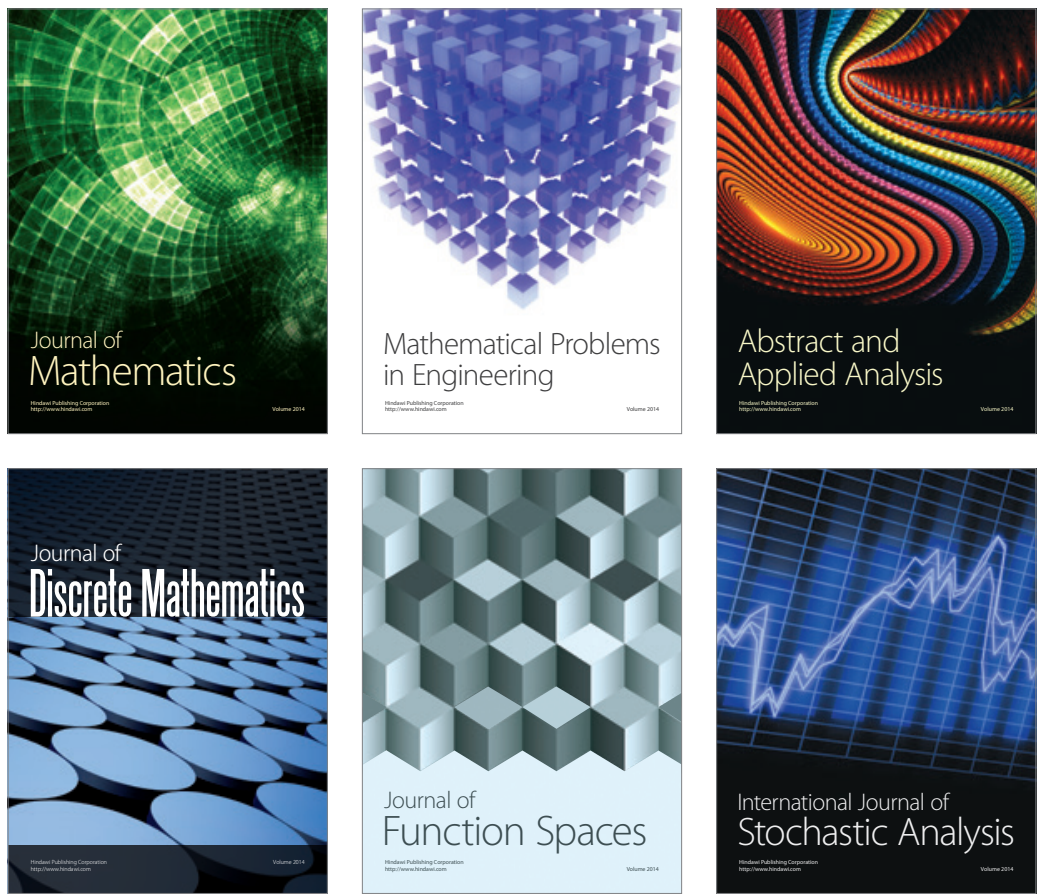

Journal of

Function Spaces

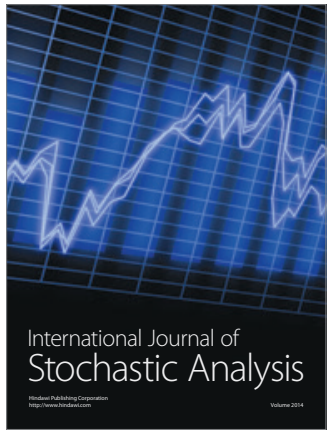

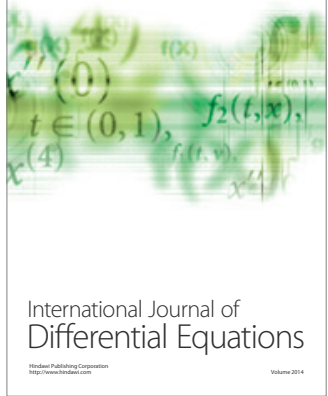
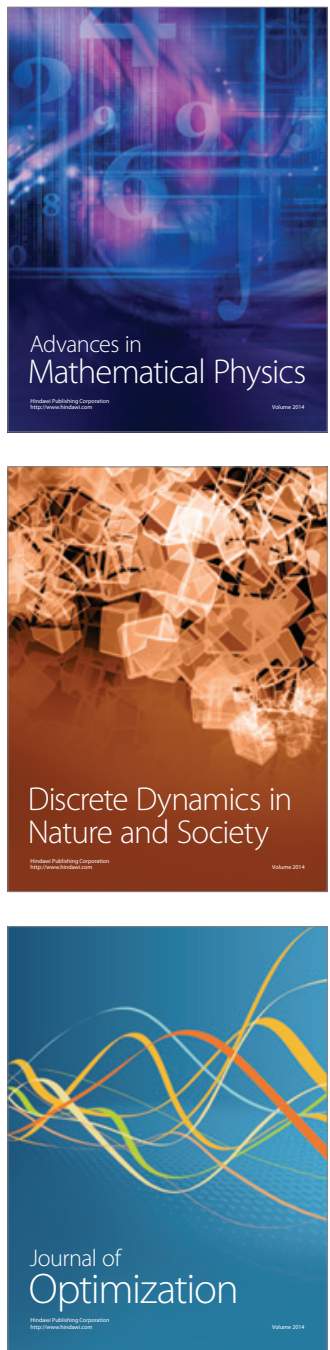